\title{
Adrenocortical Carcinoma in an Adult: Eight Months without Recurrence after Resection and Adjuvant Chemotherapy
}

\author{
Marcos Duarte Siosaki Leonardo Pelafsky Ana Tarsila Fonseca Siosaki \\ Leonardo Rufino Garcia
}

Surgical Oncology Department, Hospital Estadual Bauru, Bauru, Brazil

\section{Key Words}

Adrenocortical carcinoma · Prognosis · Recurrence · Adjuvant chemotherapy

\begin{abstract}
Adrenocortical carcinoma is a rare, aggressive malignancy that features a correspondingly poor prognosis. The disease accounts for $0.2 \%$ of all malignancies and despite multimodal therapies, its prognosis remains poor, with a mean survival of $<30$ months. This paper reports on a patient submitted to surgical resection and adjuvant chemotherapy for adrenocortical carcinoma with an ongoing follow-up of 8 months after surgery and no signs of recurrence.
\end{abstract}

(c) 2014 S. Karger AG, Basel

\section{Background}

Adrenocortical carcinoma (ACC) is a rare, aggressive malignancy that features a correspondingly poor prognosis. Its pathogenesis is poorly understood, especially at the molecular level, as the rarity of the disease renders comprehensive study difficult $[1,2]$. As a result, therapeutic options are currently limited, with medical and radiation therapy complementary to surgery, which is currently the only curative modality [1].

The disease accounts for $\leq 0.2 \%$ of all malignancies and despite multimodal therapies, its prognosis remains poor, with a mean survival of $<30$ months [3].

An overall incidence of $0.5-2$ per 1 million cases has been reported annually worldwide $[1,2]$. The disease shows a slight female gender preference and a bimodal age distribution,

Marcos Duarte Siosaki

Hospital Estadual Bauru

Avenida Luiz Edmundo Carrijo Coube, 1-100

Bauru, SP 17033-360 (Brazil)

E-Mailmarcossiosaki@yahoo.com.br 
Siosaki et al.: Adrenocortical Carcinoma in an Adult: Eight Months without Recurrence after Resection and Adjuvant Chemotherapy

with the first peak in children $<5$ years of age and the second peak in the fourth to fifth decade of life [1].

\section{Case Presentation}

A 63-year-old female patient presented left flank pain for 1 year associated with a palpated mass in this topography. In the previous 2 months, she had experienced nausea without vomiting and weight loss (not measured).

Computerized tomography was performed for staging which revealed a large mass of $20.8 \times 16.5 \times 10.6 \mathrm{~cm}\left(1,902.6 \mathrm{~cm}^{3}\right)$ in size with multiple areas of central necrosis and significant neovascularization in the left adrenal topography without a cleavage plane with pancreas and left kidney and thrombus in the inferior vena cava (fig. 1, fig. 2). No other lesions were visualized. The patient was submitted to surgery with en bloc resection of the mass, body and tail pancreatectomy, splenectomy, left nephrectomy and thrombectomy of the inferior vena cava (fig. 3, fig. 4). She was discharged from hospital on the fourth postoperative day with no complications.

Histopathology revealed an adrenal carcinoma, a solid pattern, associated with low grade, $20 \times 18 \mathrm{~cm}$ in size, and Ki67 of approximately 5\%. We observed capsule infiltration, an extensive necrosis area, clear margins and no lymph node involvement - pT4N0M0 pathologic staging IV, American Joint Committee on Cancer (AJCC) 2010, European Network for the Study of Adrenal Tumors (ENSAT) stage III. Adjuvant therapy was conducted with mitotane on an initial low-dose scheme.

After 8 months of follow-up after surgery, the patient remained without any signs of recurrence on computerized tomography and magnetic resonance scans of the abdomen.

Written informed consent was obtained from the patient for publication of this case report and any accompanying images.

\section{Discussion}

ACCs arise from cells of the adrenal cortex, which are specialized steroidogenic cells that produce glucocorticoids, mineralocorticoids, and androgens. Tumorigenesis may lead to abnormal hormone function, in approximately $60-70 \%$ of adult patients [2], and result in excess production of steroid hormones that may clinically manifest as Cushing's syndrome, Conn's syndrome, virilization or feminization [3]. However, a proportion of ACCs may be nonfunctional, and patients may present with nonspecific symptoms such as abdominal pain or fatigue [3].

Although ACC is usually sporadic, it may be associated with familial cancer susceptibility syndromes including the Li-Fraumeni syndrome, Beckwith-Wiedemann syndrome, multiple endocrine neoplasia type 1, and familial adenomatous polyposis [2]. These tumors are classified as either nonfunctional or functional depending on whether they produce corticosteroids, androgen, estrogen or mineralocorticoids. Although early studies reported that up to $50 \%$ of these were functional, more recent series have noted hormone secretion in up to $79 \%$ of ACCs [4].

Surgery is of the utmost importance in the treatment of these tumors. Open surgery with transperitoneal access is the standard treatment of all patients with localized (stage I-II) and local advanced stage (stage III) when complete resection can be achieved. Laparoscopic adrenalectomy is a safe and effective procedure for pheochromocytoma and for patients 
Siosaki et al.: Adrenocortical Carcinoma in an Adult: Eight Months without Recurrence after Resection and Adjuvant Chemotherapy

with small ACCs $(<8 \mathrm{~cm})$ without preoperative evidence of invasiveness, and adrenal masses that are judged to be only potentially malignant [5].

The resection status (R0, R1, R2) is a major predictor of prognosis for ACC. A marginfree complete resection ( $\mathrm{R} 0$ resection), in fact, provides the only means to achieve long-term survival. In order to obtain such resection it is often mandatory to resect adjacent organs such as the wall of the vena cava, liver, spleen, colon, pancreas and/or stomach [5].

Clinical stages I and II with tumors have a 5-year survival rate of 84 and $63 \%$, respectively. Stage III patients have a 5-year survival rate of $51 \%$ and stage IV drops to $15 \%$ at 1 year. It is important to know that most patients surviving at 5 years are not cured but living with the disease and $85 \%$ of patients resected for cure will develop recurrence or distant metastasis. Other factors negatively influencing the outcome are a size of $>12 \mathrm{~cm}$, high mitotic activity, necrosis, high expression of Ki67 and TP53 gene mutation positivity [6].

In 2009, a paper proposed a revision of the AJCC TNM classification, further called the ENSAT staging system [7]. The major finding of their evaluation of the proposed International Union Against Cancer (UICC) system is the observation that patients with stage II disease do not differ considerably in their disease-specific survival from patients with stage III disease. Furthermore, patients who have stage IV disease with documented distant metastases have a poorer survival than patients who have UICC stage IV disease without distant metastases, thus clearing the improving prognostic potential.

Mitotane has been the mainstay of systemic ACC treatment since the 1960s. Studies suggest that adjuvant mitotane can delay and possibly prevent a recurrence of the disease [5]. In 2008, a panel of international experts unanimously recommended adjuvant mitotane in patients with potential residual disease or a Ki67 positivity on pathologic examination of $>10 \%$. Similarly, the same panel did not consider mitotane mandatory for patients with stage I or II disease who underwent histologically proven total resection with clean margins (R0 resection) and a Ki67 index of $<10 \%$. The panel was undecided on whether to offer adjuvant therapy to stage III ACC patients following a R0 resection $[1,5]$.

\section{Conclusion}

ACC is a rare disease with poor prognosis. Although most recurrences in the adjuvant setting occur at $<6$ months postoperatively, this paper reported on a patient submitted to surgical multiorgan resection (R0 resection), pathologic stage IV (AJCC) and ENSAT stage III associated to adjuvant chemotherapy without signs of recurrence in an ongoing follow-up of 8 months.

\section{Disclosure Statement}

The authors have no conflicts of interest to disclose.

\section{References}

1 Lebastchi AH, Kunstman JW, Carling T: Adrenocortical carcinoma: current therapeutic state-of-the-art. J Oncol 2012;2012:234726.

-2 Bourdeau I, MacKenzie-Feder J, Lacroix A: Recent advances in adrenocortical carcinoma in adults. Curr Opin Endocrinol Diabetes Obes 2013;20:192-197.

3 Weissferdt A, Phan A, Suster S, Moran CA: Adrenocortical carcinoma: a comprehensive immunohistochemical study of 40 cases. Appl Immunohistochem Mol Morphol 2014;22:24-30. 


\section{Case Reports in Oncology}

\begin{tabular}{l|l}
\hline Case Rep Oncol 2014;7:222-227 & \\
\hline DOI: $10.1159 / 000362161$ & $\begin{array}{l}\text { ○ 2014 S. Karger AG, Basel } \\
\text { www.karger.com/cro }\end{array}$ \\
\hline
\end{tabular}

Siosaki et al.: Adrenocortical Carcinoma in an Adult: Eight Months without Recurrence after Resection and Adjuvant Chemotherapy

-4 Audenet F, Méjean A, Chartier-Kastler E, Rouprêt M: Adrenal tumours are more predominant in females regardless of their histological subtype: a review. World J Urol 2013;31:1037-1043.

-5 Berruti A, Baudin E, Gelderblom H, Haak HR, Porpiglia F, Fassnacht M, Pentheroudakis G: Adrenal cancer: ESMO Clinical Practice Guidelines for diagnosis, treatment and follow-up. Ann Oncol 2012;23(suppl 7):vii131-vii138.

-6 Straka M, Soumarova R, Bulejcik J, Banik M, Pura M, Skrovina M: Giant adrenocortical carcinoma with 27month disease-free survival by surgical resection alone: a case report. Biomed Pap Med Fac Univ Palacky Olomouc Czech Repub, 2013, DOI: 10.5507/bp.2013.040, Epub ahead of print.

7 Fassnacht M, Johanssen S, Quinkler M, Bucsky P, Willenberg HS, Beuschlein F, Terzolo M, Mueller H-H, Hahner S, Allolio B: Limited prognostic value of the 2004 International Union Against Cancer staging classification for adrenocortical carcinoma: proposal for a Revised TNM Classification. Cancer 2009;15:243250.

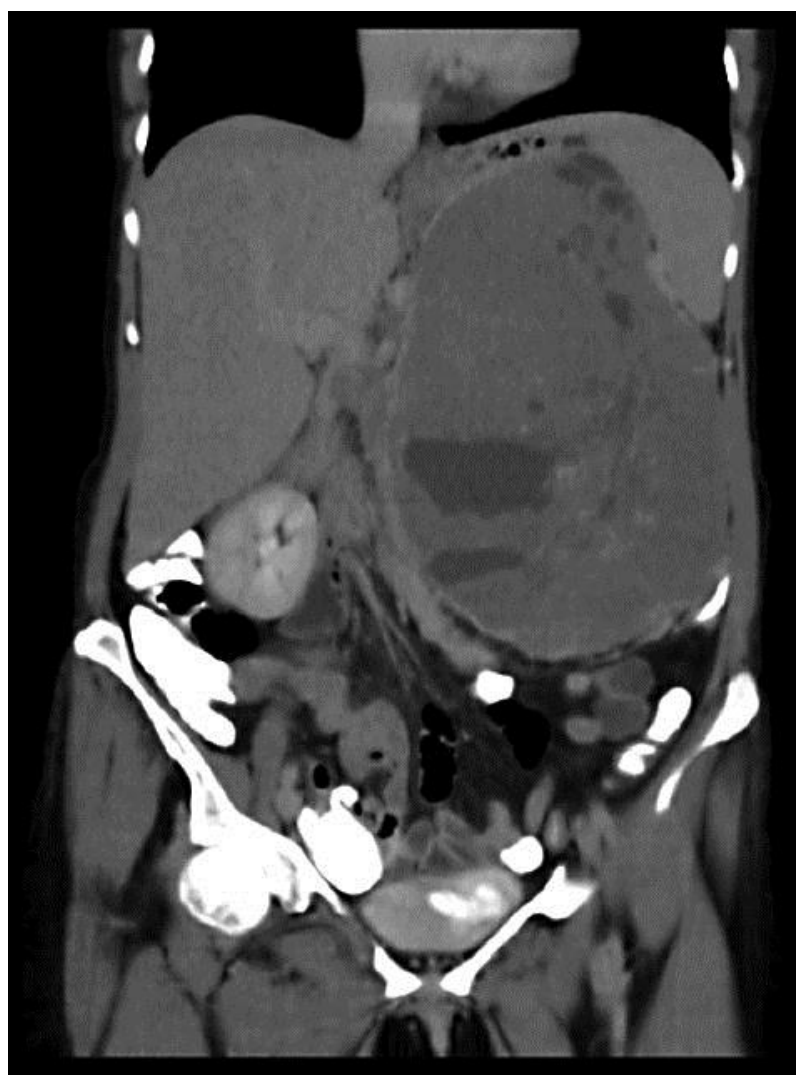

Fig. 1. Abdominal CT - coronal reconstruction - showing the mass. 


\section{Case Reports in Oncology}

\begin{tabular}{l|l}
\hline \multicolumn{2}{l}{ Case Rep Oncol 2014;7:222-227 } \\
\hline DOI: 10.1159/000362161 & $\begin{array}{l}\text { ○ 2014 S. Karger AG, Basel } \\
\text { www.karger.com/cro }\end{array}$ \\
\hline
\end{tabular}

Siosaki et al.: Adrenocortical Carcinoma in an Adult: Eight Months without Recurrence after Resection and Adjuvant Chemotherapy

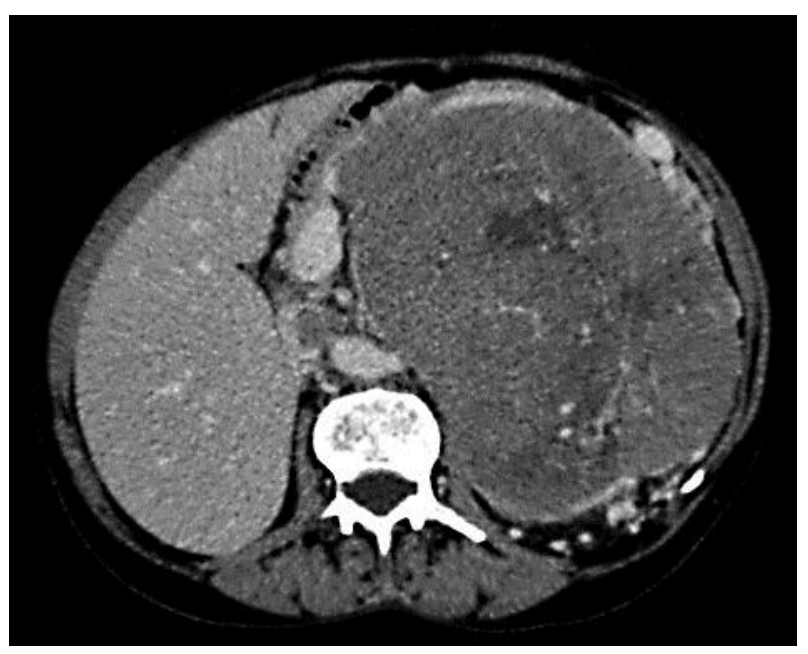

Fig. 2. Abdominal CT - horizontal reconstruction - showing the thrombus in the inferior vena cava.

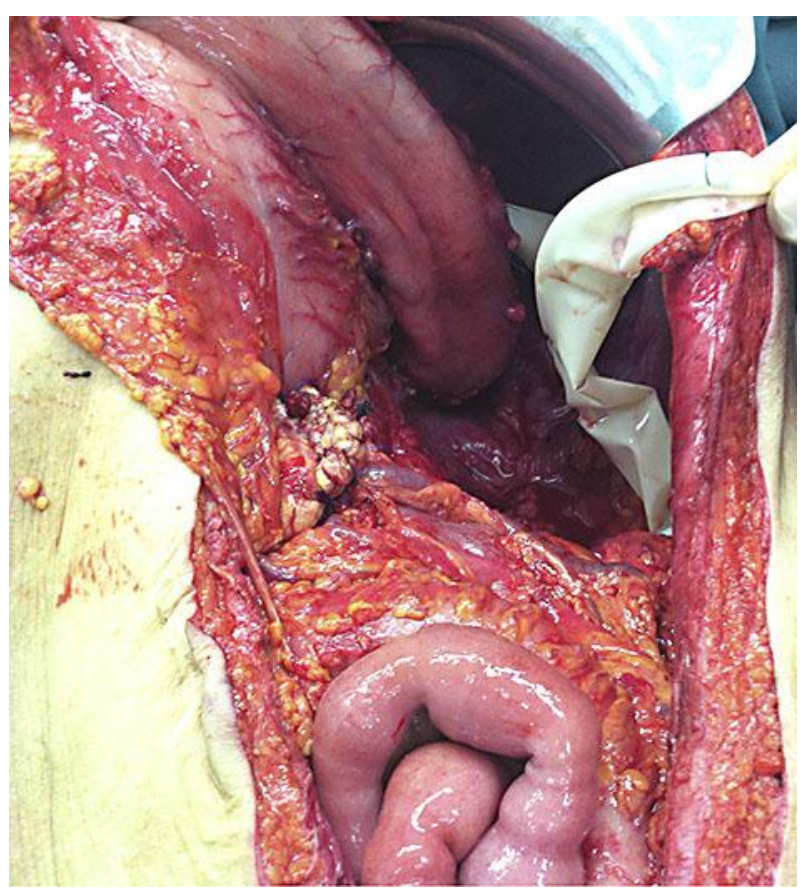

Fig. 3. Abdominal cavity after resection. 


\section{Case Reports in Oncology}

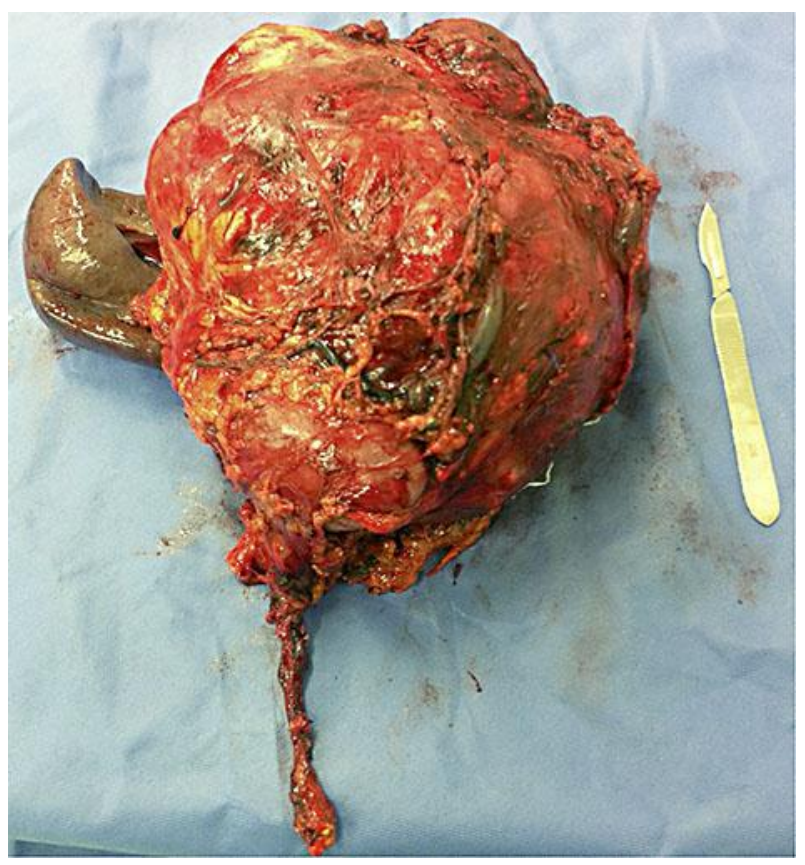

Fig. 4. Surgical specimen.
Case Rep Oncol 2014;7:222-227

DOI: $10.1159 / 000362161$

2014 S. Karger AG, Basel

www.karger.com/cro

Siosaki et al:: Adrenocortical Carcinoma in an Adult: Eight Months without Recurrence after Resection and Adjuvant Chemotherapy

- 